\title{
Phenomenological model of visual acuity
}

José A. Gómez-Pedrero

José Alonso 


\title{
Phenomenological model of visual acuity
}

\author{
José A. Gómez-Pedrero ${ }^{\mathrm{a}, \star}$ and José Alonso ${ }^{\mathrm{a}, \mathrm{b}}$ \\ a Universidad Complutense de Madrid, Applied Optics Complutense Group, Optics Department, Facultad de Óptica y Optometría, \\ C/Arcos de Jalón, 118, Madrid 28037, Spain \\ 'Indizen Optical Technologies, C/Santa Engracia 6, Madrid 28010, Spain
}

\begin{abstract}
We propose in this work a model for describing visual acuity $(V)$ as a function of defocus and pupil diameter. Although the model is mainly based on geometrical optics, it also incorporates nongeometrical effects phenomenologically. Compared to similar visual acuity models, the proposed one considers the effect of astigmatism and the variability of best corrected $V$ among individuals; it also takes into account the accommodation and the "tolerance to defocus," the latter through a phenomenological parameter. We have fitted the model to the $V$ data provided in the works of Holladay et al. and Peters, showing the ability of this model to accurately describe the variation of $V$ against blur and pupil diameter. We have also performed a comparison between the proposed model and others previously published in the literature. The model is mainly intended for use in the design of ophthalmic compensations, but it can also be useful in other fields such as visual ergonomics, design of visual tests, and optical instrumentation. ๑ The Authors. Published by SPIE under a Creative Commons Attribution 3.0 Unported License. Distribution or reproduction of this work in whole or in part requires full attribution of the original publication, including its DOI. [DOI: 10.1117/1. JBO.21.12.125005]
\end{abstract}

Keywords: visual acuity; defocus; model fitting; ophthalmic optics.

Paper 160492RR received Jul. 14, 2016; accepted for publication Nov. 17, 2016; published online Dec. 14, 2016.

\section{Introduction}

High contrast visual acuity stands out as a good metric for assessing the optical quality of the eye. It is routinely measured at the prescription room, it only involves one parameter, and it presents strong correlation with defocus and/or astigmatism, which are the aberrations (along with transverse chromatic aberration) that really hinder the optical quality of the ametropic eye or the lens-eye system $^{1,2}$ in the case of ametropic compensated eyes. In the case of ophthalmic lenses, these second-order aberrations appear at oblique gaze directions for constant power lenses or spread around a large portion of the lens for variable power lenses. For healthy eyes without abnormal amounts of high order aberrations (HOA), and for defocus and astigmatism larger than about $0.75 D$, blur and pupil size will mainly determine the visual acuity. ${ }^{3-5}$ However, for small values of second-order aberrations (defocus and astigmatism), visual acuity will depend on the complex interactions among all the factors affecting it.

The relationship between visual acuity $(V)$ and blur has been comprehensively studied through the 20th century in many studies. Some of them were made with a reduced number of individuals under controlled conditions, and their goals were to relate the $V$ with blur and other relevant parameters, such as the pupil diameter, accommodation, and presence of HOA. Other studies involved large samples of individuals, typically measuring the unaided $V$ as a function of the refractive error.

Atchison et al. ${ }^{6}$ measured the monocular unaided visual acuity of 22 subjects with dilated pupils $(0.5 \%$ tropicamide $)$ by using artificial pupils with either constant retinal illuminance or constant chart illuminance. In this study, the refractive errors were all myopic ranging between -0.75 and $-7.5 \mathrm{D}$. Holladay et al. $^{5}$ compiled measurements from 12 previous studies to

*Address all correspondence to: José A. Gómez-Pedrero, E-mail: jagomezp@ ucm.es obtain a reference grid of $V$ versus myopic defocus and pupil diameter.

Bradley et al. ${ }^{7}$ measured visual acuity of four individuals against artificially induced spherical and astigmatic blur. Two of the participants of this study had their accommodation blocked with tropicamide to test how accommodation helps keep high acuity when a low amount of negative defocus is present.

Villegas and $\mathrm{Artal}^{1}$ measured the visual acuity of a user of a progressive power lens when looking through different points on the lens and compared the obtained values with the coefficients of the Zernike polynomial expansion of the wavefront of the isolated lens and the lens-eye system. They also compared $V$ with different metrics computed from the wavefront. These authors found that, at the regions located at both sides of the progressive corridor, the predominant factor in the loss of $V$ was second-order astigmatism (the spherical defocus was corrected through the study) as its contribution to the wavefront error was an order of magnitude higher than that of the HOA.

Applegate et al. ${ }^{8}$ measured acuity by convoluting the optotypes with a point spread function (PSF) corresponding to isolated modes of Zernike polynomials. They found a linear variation of $V$ with the amount of aberration coefficients, and the larger slopes were found to be those of second-order polynomials (corresponding to defocus and astigmatism).

Among the studies with a large numbers of participants, Pincus ${ }^{9}$ measured and tabulated the unaided visual acuity of a population of around 7600 young subjects was. Another classical study on the relationship between refractive blur and visual acuity is the Orinda Vision Study reported by Peters. ${ }^{10}$ In this work, the unaided visual acuity of a population of around 7200 subjects was measured. The differences between these two works are that in Peters's, the sample were divided into three age groups, and no drugs were used to block accommodation or dilate the pupil. As $V$ presents significant differences 
among individuals, even under similar conditions, those studies made with large samples provide meaningful information about the relation between mean visual acuity and spherical and astigmatic blur.

In addition to the measurements ${ }^{3}$ and clinical studies, some acuity models have been proposed in the literature. Legge et al. ${ }^{4}$ proposed an inverse relationship between $V$ and defocus after measuring the Snellen visual acuity of four subjects. This model was further refined by Smith, ${ }^{11}$ after analyzing a number of classical clinical studies and models on refractive error and visual acuity. Smith's model is also based on an inverse relationship between $V$ and the product of defocus and pupil diameter, as would be expected from a geometrical analysis of the problem. Smith also proposes a functional form that sets $V$ equal to one when the geometrical blur tends to zero. Neither Legge et al. ${ }^{4}$ nor Smith ${ }^{11}$ considered the effect of astigmatism in their models. Raasch ${ }^{12}$ proposed the quantification of secondorder blur (including spherical defocus and astigmatism) as the modulus of the optometric vector defined by Thibos. ${ }^{13}$ Raasch established the relationship of this second-order blur with $V$ through an empirical equation with coefficients obtained by fitting Pincus' data. In this model, neither accommodation nor pupil size are considered. Blendowske ${ }^{14}$ recently presented a visual acuity model that uses the astigmatic blur proposed by Raasch and takes into account the visual acuity obtained with the best possible correction, $V_{b c}$. Otherwise, the model has no other free parameters.

More refined models have been proposed. Greivenkamp et al. ${ }^{15}$ modeled the acuity in a series of schematic eyes by the numerical computation of the PSF of those schematic eyes through exact ray tracing. Those results have been generalized by Nestares et al. ${ }^{16}$ in a model that takes into account the individual aberrations of the eye under polychromatic light and the visual processing of the retinal image. This model also incorporates a Bayesian classifier to predict the acuity of a particular subject from the clinical data available. This is probably the most comprehensive model of visual acuity to date as it takes into account not only optical factors but also the filtering process carried out at neural levels. Assuming that all the required data and enough computing power are available, Nestares et al.'s model would probably be the more complete choice.

Close examination of the available experimental data and models shows that accurate prediction of visual acuity is a formidable task. First, because of its subjective nature and the multitude of factors affecting it, measurement of visual acuity is intrinsically inaccurate. For example, if we consider the asymptotic dependence of the mean acuity of large groups of people with large values of defocus and compare the measurements by Pincus and Peters, we find very different behaviors. The Pincus's acuity measured values getting smaller much faster than Peters's, most surely because of the dilation of the pupils in Pincus' measurements. A model in which $V$ is proportional to a power of the size of the blur patch with an exponent larger than one could fit Pincus's data well but would fail to properly fit Peters's. Second, even if we restrict ourselves to measurements on the same person and with identical procedure, acuity values may change significantly from one experiment to other, depending on the status of the patient.

Despite these difficulties in modeling visual acuity, we still consider a pseudophenomenological approach to model its behavior with respect to some critical parameters to be a great help in some fields, in particular for the design of optical compensations for refractive errors. It could also be employed in other applications such as visual ergonomics, design of visual tests, analysis of optical instruments aimed to be used by the eye (binoculars, microscopes, and so on), and virtual reality. Our aim is then to provide a model that would depend on simple, easy to measure, and readily available parameters, and that would provide the right behavior of the visual acuity as these parameters change from person to person. Also, we would like the model to be well behaved with respect to averages: as we compute the mean of the predicted acuity across a population in which the parameters may change, we would expect the model to provide a better prediction of clinically measured and averaged $V$ values.

The description of this model, the way we tune its parameters, and the results we obtained with it are presented in this paper.

\section{Materials and Methods}

\subsection{Visual Acuity Model}

Let us consider an eye with refractive error at the plane of the entrance pupil $\mathbf{R}=[S, C \times \theta]$, with $S$ and $C$ being the sphere and cylinder, respectively, and $\theta$ being the orientation of the cylinder axis. In terms of the eye aberrations, refractive error is given by the second-order aberrations of the eye. ${ }^{17}$ Therefore, if a planar wavefront arrives at the eye's entrance pupil, and we describe the aberrated wavefront at the eye's exit pupil as a series of Zernike polynomials, then it is well known ${ }^{17}$ that the coefficients of the second-order polynomials, namely $c_{2,-2}$, $c_{20}$, and $c_{22}$, are related to the refractive error $[S, C \times \theta]$ through the following equations: ${ }^{17} c_{2,-2}=\frac{1}{16 \sqrt{6}} D^{2} C \sin 2 \theta$, $c_{22}=\frac{1}{16 \sqrt{6}} D^{2} C \cos 2 \theta$, and $c_{20}=-\frac{1}{32 \sqrt{3}} D^{2}(2 S+C)$, where $D$ is the diameter of the entrance pupil of the eye. We may also describe the refractive error as the curvature of the wavefront at the entrance pupil of the eye when this wavefront produces a well-focused image on the retina. The matrix expression for this curvature is the dioptric power matrix described elsewhere, ${ }^{18}$

$$
\begin{aligned}
\mathbb{R} & =\left[\begin{array}{cc}
S+C \sin ^{2} \theta & -C \cos \theta \sin \theta \\
-C \cos \theta \sin \theta & S+C \cos ^{2} \theta
\end{array}\right] \\
& \equiv-\frac{16 \sqrt{6}}{D^{2}}\left[\begin{array}{cc}
\sqrt{2} c_{20}+c_{22} & \frac{c_{2-2}}{2} \\
\frac{c_{2-2}}{2} & \sqrt{2} c_{20}-c_{22}
\end{array}\right]
\end{aligned}
$$

A straightforward geometrical computation reveals that the blur patch on the retina has an elliptical shape with an average size that is proportional to the norm of the refractive error matrix when diffraction and HOA are neglected. This blur, $\delta=\|\mathbb{R}\|$, which coincides with the vector length defined by Thibos et al. ${ }^{13}$ and used by Raasch, ${ }^{12}$ can be expressed in terms of the spherocylindrical components of the refractive error as

$\delta=\frac{1}{2} \sqrt{4 H^{2}+C^{2}}$,

where $H=S+C / 2$ is mean curvature of the wavefront, i.e., the spherical equivalent. The blur just described corresponds to the myopic, hyperopic, and/or astigmatic condition of the subject. This blur, however, can be modified by placing a compensating device before (ophthalmic and contact lenses) or inside the eye 
(intraocular lenses). The resulting refractive error can be written as $\mathbb{R}^{*}=\mathbb{R}-\mathbb{P}$, where $\mathbb{R}$ is the uncompensated refractive error of the individual and $\mathbb{P}$ is the dioptric power matrix corresponding to the compensation. Normally, the compensating device would be designed so that $\mathbb{P}=\mathbb{R}$ and hence $\mathbb{R}^{*}=0$. But in many cases, the compensating device fails to fully compensate the refractive error at the whole field of view, in which case a nonzero resulting refractive error and its corresponding blur will be present. Predicting $V$ in such circumstances would be useful for evaluating the performance of the compensating device.

According to Smith, ${ }^{11}$ for high enough amounts of blur (around $1 D$ ), the minimum angle of resolution (MAR) is directly proportional to both pupil diameter and blur, i.e., MAR $\propto D \delta$. The MAR is defined, according to the classical definition of resolution, as the angular separation between two object points whose blurred images overlap on the retina but still can be perceived as distinct ones. This tolerable overlapping (that may change among individuals) determines the proportionality constant between MAR and $D \delta$, so following Smith, we will write

$$
\mathrm{MAR}=K D \delta
$$

The definition of blur (2), and hence the MAR given by Eq. (3), does not depend on the cylinder axis. Nevertheless, if both $\mathbb{R}$ and $\mathbb{P}$ represent astigmatic wavefronts, the blur will depend on the relative orientation between them.

Equation (3) fails when the residual refractive error goes toward zero. Smith proposed the mathematical correction

$$
\mathrm{MAR}=\sqrt{1+(K D \delta)^{2}}
$$

which asymptotically behaves as Eq. (3) for large residual refractive errors but smoothly forces the MAR toward $1^{\prime}$ when the defocus goes toward zero. That is an interesting idea, but it could be too rigid as it is precisely the transition region between the minimum MAR $\left(1^{\prime}\right)$ and the asymptotic behavior that is more interesting to model because that is the region in which the subject clearly notices a drop of visual performance (e.g., near the boundaries of the visual field of a progressive lens). Also, minimum MAR is locked to $1^{\prime}$ and that cannot account for the variability of $V_{b c}$ that we find across any population. Using a similar idea as Blendowske's, ${ }^{14}$ incorporating $V_{b c}$ into the model, we propose the next expression for the MAR

$$
\mathrm{MAR}=\left\{\left[\operatorname{MAR}_{0}(D)\right]^{q}+(K D \delta)^{q}\right\}^{1 / q},
$$

where $\operatorname{MAR}_{0}(D)$ is the minimum resolution angle achieved by the best corrected eye, which is dependent on pupil size, and $q$ is a parameter that controls the speed at which the eye increase the MAR from the minimum value to the asymptotic one as the blur increases. In other words, $q$ describes the tolerance to defocus of the eye we want to model. When the defocus is large, $\operatorname{MAR}_{0}(D)$ can be neglected, and then we recover Eq. (3) as an asymptotic behavior of Eq. (5). When the defocus goes toward zero, MAR tends to $\operatorname{MAR}_{0}(D)$. Large values of $q$ would make the MAR more insensitive to small defocus, but then the transition to the asymptotic behavior would be faster. Small values of $q$ would make the model more sensitive to small defocus, with a slower transition to the asymptotic regime. It is interesting to notice that, as said before, for large values of the blur, MAR $\approx K D \delta$ according to Eq. (5). However, some works ${ }^{4}$ predict other functional dependence for the MAR against large blurs, typically, MAR $\sim \delta^{w}$. This dependence could be easily incorporated to the proposed model by changing Eq. (5) accordingly, but we have preferred to keep the number of parameters as low as possible.

To use the model, we need a functional form for $\operatorname{MAR}_{0}(D)$. Here, we could either use Atchison et al.' $s^{6}$ or Holladay et al.' $s^{5}$ tables of MAR versus defocus at best correction. We selected Holladay et al.'s as their data are obtained as an average from many different experiments. For small values of the pupil diameter, the MAR presents the inverse dependence characteristic of diffraction. As the pupil diameter increases, the MAR first reaches a minimum value (for a pupil diameter of around $3 \mathrm{~mm}$ ) and then increases at a slow rate due to the presence of HOA. Finally for pupil sizes larger than $6 \mathrm{~mm}$, the MAR stabilizes as a consequence of the Stiles-Crawford effect. We have modeled this behavior with a two-term function $\operatorname{MAR}_{H}(D)$, the first one accounting for the inverse dependence on pupil size for small pupils and the second one a sigmoid type function, negligible for small pupils and growing to a stationary value for larger ones

$\operatorname{MAR}_{H}(D)=\frac{a}{D}+b\left(1-\frac{1}{1+c D^{3}}\right)$,

where $D$ is the pupil diameter and $a, b$, and $c$ are fitting parameters. Many other functions can be used to reproduce the experimental behavior, but the one proposed in Eq. (6) provided the best statistical results with the smallest number of parameters. The minimum value of $\operatorname{MAR}_{H}(D)$ is 0.45 arc min (corresponding to a $V$ of 2.2 in decimal scale or $-0.34 \operatorname{LogMAR}$ ), and this value is found for a pupil diameter of $3 \mathrm{~mm}$. However, we want to account for the variability of the peak $V$ among different individuals, so we will define the individual minimum resolution angle as

$\operatorname{MAR}_{0}\left(D, V_{b c}\right)=\operatorname{MAR}_{H}(D)-0.45+V_{b c}^{-1}$,

which is the fit to Holladay et al.'s data vertically displaced so that its minimum value matches the minimum of the individual whose $V$ we want to model. This is given by the best corrected visual acuity, $V_{b c}$, which is routinely measured in a typical eye examination and accounts for some of the nonparaxial, retinal, and neural effects on the $V$ versus defocus model of an individual. More details about the fitting of $\operatorname{MAR}_{H}(D)$ can be found in the results section (see Fig. 1).

The final thing to take into account is the effect of accommodation. This parameter is not considered in previous models although its effect on visual acuity when positive refractive errors are induced in young individuals is well known. ${ }^{7}$ For example, if we want to predict the loss of visual acuity due to the effect of oblique aberrations and/or unwanted astigmatism and sphere error when looking through an ophthalmic lens, we should take into account the capacity of the eye to accommodate and compensate for positive errors, as said before. As expressed in Eq. (5), MAR is a monotonically growing function of blur, so accommodation should minimize $\delta$ to improve $V$. We can also assume that the effect of accommodation $A$ is to simply change the spherical component of the refractive error 


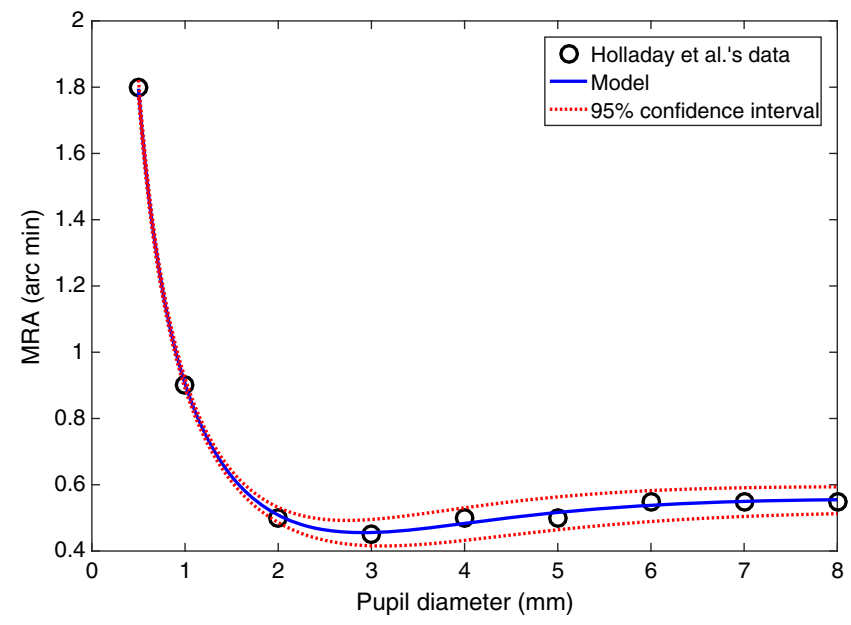

Fig. 1 Best corrected (zero blur) MAR as a function of pupil diameter as given by Holladay et al. (circles), and fit of these data to the model function $\operatorname{MAR}_{H}(D)$ plotted as a continuous line (blue in color). The $95 \%$ confidence interval of the model is also plotted as two dashed lines (red in color).

$\delta=\frac{1}{2} \sqrt{4(H-A)^{2}+C^{2}}$.

The accommodation value that minimizes Eq. (8) is just the spherical equivalent $H$, but only for positive errors and provided this spherical equivalent is lower than the subject's amplitude of accommodation $A_{\max }$. Therefore, we will take the accommodation $A$ as

$A=\left\{\begin{array}{ll}0, & H<0 \\ A_{\max }, & H \geq A_{\max } \\ H, & 0<H<A_{\max }\end{array}\right.$.

This assumption is equivalent to the accommodation trying to bring the circle of least confusion onto the retina. We know that in the presence of astigmatism, $V$ will depend on the shape of the object and the orientation of the principal directions of the resulting refractive error $\mathbb{R}^{*}$. Taking this effect into account would force us to use another expression for $\delta$ that would depend on the orientation of the cylinder axis. As there are no conclusive results about the relation between $V$ and cylinder orientation axis, ${ }^{19}$ we will stick to the standard blur given by Eq. (8).

In summary, the proposed model estimates the $V$ of an individual's eye as a function of its pupil size and defocus

$$
V(D, \delta)=\frac{1}{\left\{\left[\operatorname{MAR}_{0}(D)\right]^{q}+(K D \delta)^{q}\right\}^{1 / q}},
$$

where $\delta$ is given by Eq. (8). The model requires two easily available parameters: the maximum monocular acuity achieved by the individual, $V_{b c}$, and the amplitude of accommodation, $A_{\max }$. It also requires two more parameters, $K$ and $q$, that are not measured in a typical eye exam. To obtain meaningful values for them, we could fit the model to some experimental measurements of the individual's $V$, or we could use averaged values from $V$ measurements in large population groups. In $\log$ MAR scale, more commonly employed in clinical research, the model reads $\log \operatorname{MAR}=\frac{1}{q} \log _{10}\left[\operatorname{MAR}_{0}(D)^{q}+(K D \delta)^{q}\right]$.

The logMAR scale reflects the correct psychophysics approach to describe visual acuity when it is measured with optotypes whose size changes in geometrical (exponential) progression. Because of this, $\log$ MAR scale allows for direct average of $V$ from optotypes in different charts. Although decimal scale could be less common for clinical researchers, we will use it for fitting and statistical analysis, as it is better mathematically behaved and the significant behavior difference between both scales appears at very low $V$; further, we are mainly interested in the modeling of high visual acuity.

\section{Results and Discussion}

In Fig. 1, we first show the fit of the function $\operatorname{MRA}_{\mathrm{H}}(D)$ to Holladay et al.'s data, as it is part of the $V$ model. As stated, Holladay et al. compiled measurements from 12 previous studies to obtain a reference grid of $V$ versus myopic defocus and pupil diameter. To obtain the fitting parameters in $\operatorname{MRA}_{H}(D)$, we have used only the data for zero defocus.

The fitting of Eq. (6) to Holladay et al.'s data, when $D$ is measured in millimeters and $\mathrm{MRA}_{H}$ in arcminutes, are: $a=0.897 \pm 0.013(0.884,0.910) \mathrm{mm}, b=0.49 \pm 0.03(0.462$, $0.524)$ arcmin, and $c=0.017 \pm 0.004(0.013,0.021) \mathrm{mm}^{-3}$, where the error values are given by the boundaries of $95 \%$ confidence level.

As shown in Fig. 1, the model fits the experimental data well, the fit residuals presenting a standard deviation of 0.017 . Had we wish to customize the model for a given individual, it would be necessary to measure the visual acuity for different pupil sizes and fit the model given by Eq. (6) to those data.

To check the consistency of the whole model, we used the function $\operatorname{MAR}_{H}(D)$ with the coefficients thus obtained to fit the remaining Holladay et al.'s data, i.e., the values of $V$ with respect to nonzero blur and pupil size. To compare with existing models, we have selected Smith's as it has a dependency with both blur and pupil diameter. However, in its original form, Smith's model will always predict maximum $V$ equal to 1 ; hence, it will not properly fit Holladay et al.'s data for which maximum $V$ is far greater than 1. To make a fair comparison, we have then modified Smith's model as

$V_{S}(D, \delta)=\left[V_{b c}^{-2}+(K D \delta)^{2}\right]^{-1 / 2}$,

$V_{b c}$ being now the maximum uncorrected visual acuity predicted by the model.

The results of the fitting are shown in Fig. 2, where we have plotted the curves of $V$ obtained with the two models against blur for six different pupil sizes, and in Table 1, where we show the values of the model parameters and their $95 \%$ confidence intervals. It is important to notice that, for the proposed model, function $\operatorname{MRA}_{\mathrm{H}}(D)$ is fixed, and the only fitting parameters are $K, q$, and $V_{b c}$. For Smith's model, the fitting parameters are the coefficients $K$ and $V_{b c}$.

The overall fitting quality of the proposed model to Holladay et al.'s data is good for all diameters except $D=1 \mathrm{~mm}$, while modified Smith's model fails for all diameters in the low-blur region. This suggests that the incorporation of the $q$ parameter and the dependence of $V_{b c}$ with pupil diameter are significant to properly describe the behavior of $V$ against blur and pupil diameter. 

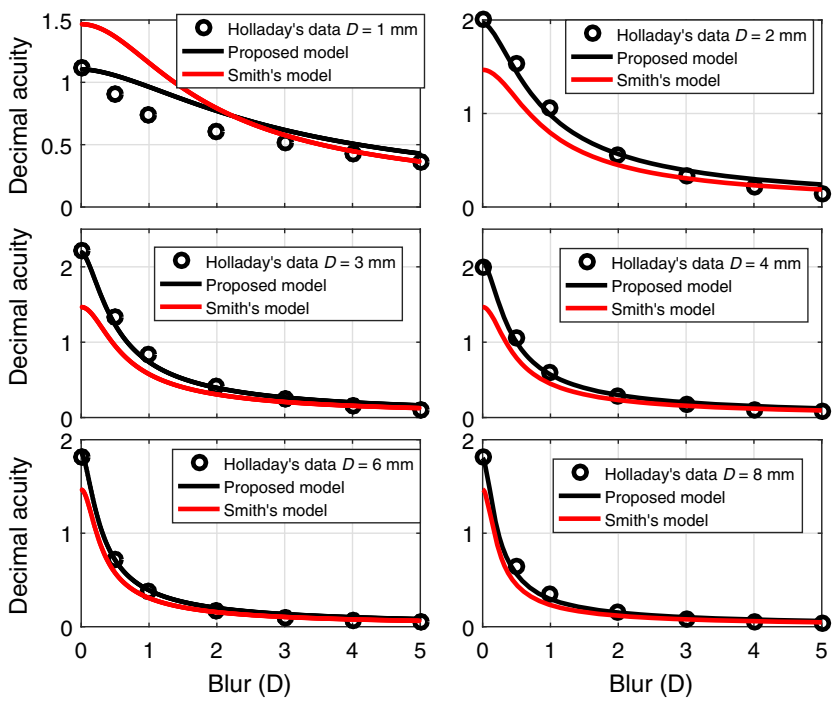

Fig. 2 Model fit to the complete set of data given by Holladay et al. (circles). We have represented the $\mathrm{V}$ against blur for different pupil sizes; the dark curves (black in color) are the results obtained after applying our model using the coefficients of $\operatorname{MAR}_{H}(D)$ with the parameters obtained through the fit shown in Fig. 1. The light (red in color) curves represents the results obtained after fitting the modified Smith's model proposed in Eq. (12) to the same data set.

The values of the coefficients obtained along with the $95 \%$ confidence interval are presented in Table 1. We have also included a set of goodness-of-fit statistical indicators. These are the adjusted $R^{2}$ coefficient, the root mean square error (RMSE) of the residuals, and the coefficients Akaike indicator coefficient (AIC) and Bayesian indicator coefficient (BIC) based on information theory, which are more useful to compare the quality of fitting of different models to the same dataset. ${ }^{20}$ For the proposed model, both AIC and BIC are much lower than the values obtained for the modified Smith's model, which indicates that the proposed model is a better tool for fitting Holladay et al.'s data. This is corroborated by the values of both $R_{\text {adj }}^{2}$ and RMSE.

We have also compared the proposed model with those of Raasch's ${ }^{12}$ and Blendowske's ${ }^{14}$ by fitting to the data reported by Holladay et al. However, as those models lack an explicit dependence with pupil diameter, we have fitted separately the data corresponding to different pupil sizes to make a fair comparison. Obviously, this would mean that the fitting parameters will show a dependence with the pupil diameter (ideally not for the proposed model). Blendowske's model ${ }^{14}$ only has one parameter and is defined by the expression

$V_{B}(\delta)=\frac{V_{b c}}{1+\delta^{2}}$.
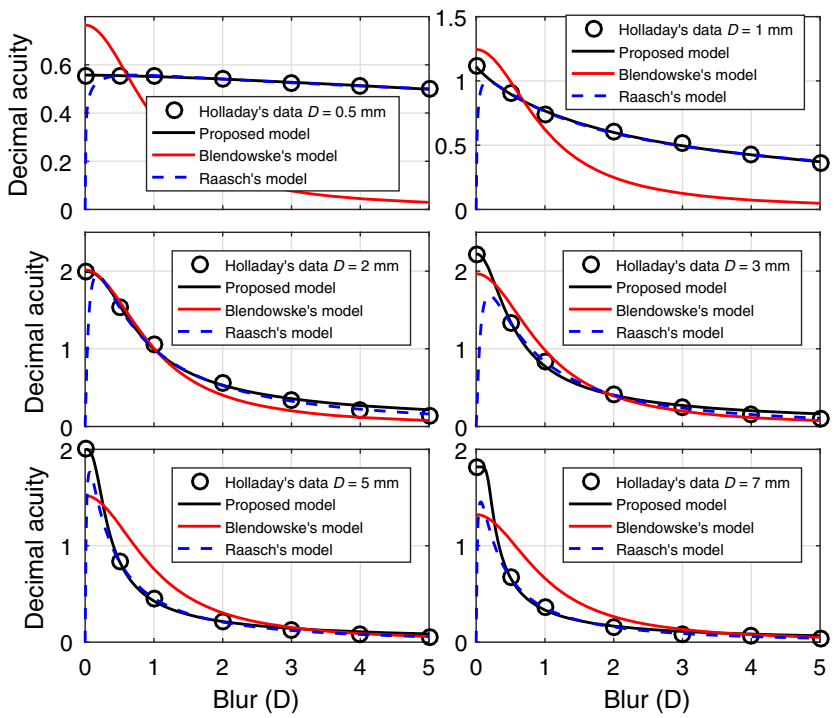

Fig. 3 Model fit to the complete set of data given by Holladay et al. (circles) taken pupil by pupil so that each panel represents the fit of the models to the values of Holladay et al.'s $\mathrm{V}$ corresponding only to this pupil diameter. We have represented the $\mathrm{V}$ against blur for different pupil sizes; the dark curves (black in color) are the results obtained after applying our model using the coefficients of $\operatorname{MAR}_{H}(D)$ with the parameters obtained through the fit shown in Fig. 1. The light (red in color) curves represents the results obtained after fitting Blendowke's model, and the dashed curves (blue in color) represent the fitting of the data to Raasch's model.

Raasch's model, when expressed in terms of decimal visual acuity, is defined as

$V_{R}(\delta)=10^{-\left[A_{0}+A_{1} \log _{10}(\delta)+A_{2} \log _{10}^{2}(\delta)\right]}$,

where $A_{0}, A_{1}$, and $A_{2}$ are the model parameters that lack a clear physiological meaning.

We show the results obtained for the pupil diameters of 0.5 , 1, 2, 3, 5, and $7 \mathrm{~mm}$ in Fig. 3. As in the previous analysis, we omit pupil diameters 6 and $8 \mathrm{~mm}$ to avoid cluttering the figures and because the three models behave very similarly at these diameters as they do for 5 and $7 \mathrm{~mm}$. As it can be seen in this figure, the proposed model correctly fits the data for all the diameters considered. Blendowske's model fits correctly the data corresponding to medium-sized pupils ( 2 and $3 \mathrm{~mm}$ ). For small pupils it fails for all blur values, whereas for large pupils it seems to provide good prediction for large values of blur.

In turn, Raasch's model fits quite well the visual acuity for moderate to large blur for each pupil, but it is not able to correctly predict $V$ for low values of blur. This is due to the mathematical properties of the fitting function. In terms of decimal acuity, it tends to zero as the blur goes to zero. In terms of

Table 1 Value of fit parameters and statistical indicators of goodness-of-fit for the fit of Holladay et al.'s data with the proposed and Smith's models. Legend: $R_{\text {adj }}^{2}$, adjusted $R^{2}$ coefficient; RMSE, root mean square error of the residuals; AIC, Akaike information coefficient; BIC, Bayesian information coefficient. The limits of the $95 \%$ of the confidence interval for the fitting parameters are expressed as error limits.

\begin{tabular}{lcccccccc} 
Model & $V_{b c}$ & $K$ & $q$ & $R_{\mathrm{adj}}^{2}$ & RMSE & AIC & AICc & BIC \\
\hline Proposed & $2.20 \pm 0.06$ & $0.41 \pm 0.04$ & $1.7 \pm 0.3$ & 0.98885 & 0.062 & -168.07 & -167.66 & -161.64 \\
Smith & $1.47 \pm 0.18$ & $0.53 \pm 0.14$ & $\mathrm{n} / \mathrm{a}$ & 0.71834 & 0.314 & 34.42 & 34.32 & 38.71 \\
\hline
\end{tabular}


Table 2 Values of the fitting coefficient together with the 95\% confidence interval (expressed as errors) obtained after fitting the proposed model and those of Blendowske's and Raasch's to Holladay et al.'s acuity data separately for each value of pupil diameter.

\begin{tabular}{|c|c|c|c|c|c|c|c|}
\hline \multirow{2}{*}{$\frac{\text { Model }}{D(\mathrm{~mm})}$} & \multicolumn{3}{|c|}{ Proposed } & \multirow{2}{*}{$\frac{\text { Blendowske }}{V_{b c}}$} & \multicolumn{3}{|c|}{ Raasch } \\
\hline & $V_{b c}$ & $K$ & $q$ & & $A_{0}$ & $A_{1}$ & $A_{2}$ \\
\hline 0.5 & $2,21 \pm 0,09$ & $0,23 \pm 0,15$ & $1,49 \pm 0,50$ & $0.76 \pm 0,34$ & $0.25 \pm 0.29$ & $0.02 \pm 0.41$ & $0.06 \pm 0.87$ \\
\hline 1.0 & $2.23 \pm 0.20$ & $0.32 \pm 0.10$ & $0.89 \pm 0.17$ & $1.24 \pm 0,130$ & $0.12 \pm 0.28$ & $0.30 \pm 0.05$ & $0.20 \pm 2.10$ \\
\hline 2.0 & $2.23 \pm 0.20$ & $0.46 \pm 0.09$ & $2.46 \pm 1.43$ & $2.01 \pm 0.08$ & $1.15 \cdot 10^{-10} *$ & $0.77 \pm 0.32$ & $0.51 \pm 2.31$ \\
\hline 3.0 & $2.22 \pm 0.14$ & $0.41 \pm 0,09$ & $2.18 \pm 1.47$ & $1.97 \pm 0.08$ & $0.07 \pm 0.62$ & $0.86 \pm 1.23$ & $0.60 \pm 6.80$ \\
\hline 5.0 & $2.12 \pm 0.10$ & $0.43 \pm 0.08$ & $2.45 \pm 6.72$ & $1.66 \pm 0.08$ & $0.21 \pm 1.20$ & $0.93 \pm 2.44$ & $0.47 \pm 13.5$ \\
\hline 7.0 & $2.28 \pm 0.10$ & $0.47 \pm 0.07$ & $3.03 \pm 1051$ & $1.52 \pm 0.13$ & $0.34 \pm 1.48$ & $1.00 \pm 3.10$ & $0.42 \pm 523$ \\
\hline
\end{tabular}

$\log$ MAR acuity, it diverges. We have to assume that Raasch's model was intended for describing the loss of $V$ at medium to large blur.

The values obtained for the fitting parameters are given in Table 2. Regarding the proposed model, both $V_{b c}$ and $K$ present stable values (and they also have a reduced interval of confidence), as is expected given the dependence of this model with the pupil diameter. The coefficient $q$ is not so well behaved, as it varies between 0.89 and 3.03 with increasing confidence interval for high pupil sizes. This could be due to the fact that $q$ affects the shape of the $V$ curve mainly for blur values smaller than $1 D$. Holladay et al.'s data only provide two points per curve in this interval, so there is probably not enough information to reduce the uncertainty on $q$.

In the case of Blendowske's and Raasch's models, the data collected in Table 2 show a strong variation of the fitting parameters with the pupil size, as is expected by the nature of these models. It is particularly interesting that, for Raasch's model, the values of both $A_{1}$ and $A_{2}$ increase with the pupil size (albeit due to the high width of the confidence interval, it is difficult to state this conclusively), which may hint a possible way to introduce pupil size dependence in this model.

In Table 3, we present the values of the coefficients that indicate the goodness-of-fit for all the pupils considered. Now, with respect to the coefficients listed in Table 2, we have added AICc, which is a variant of AIC corrected for small sample sizes, and wAIC, which is the weight of evidence that indicates the probability that a given model represents the true fit to the data when compared to other models for the same dataset. $^{20}$ The proposed model presents the highest value of wAIC for all the pupil sizes considered, so it statistically represents the best choice (this is emphasized by the fact that AIC, AICc, and BIC reach minimum value for the proposed model for all pupil sizes). Raasch's model is handicapped by its behavior at zero blur. Had we removed the first point in each curve, its statistics would had improved. Nevertheless, we have kept the zero blur points as the predictions for low blur values are important to us.

We have also tested the proposed model with a larger data set, for which we have selected the data provided by Peters that were obtained within the Orinda Vision Study. ${ }^{10}$ Those data are distributed into three age groups and the population sample includes hyperopes, so it is possible to check the effect of accommodation on the visual acuity as predicted by our model (8). To do so, we have to make use of the accommodation parameter, $A_{\max }$, introduced in Eqs. (8) and (9).

Peters's data are represented as acuity iso-lines in the spherecylinder plane. These kinds of representations should comply with the spherocylindrical transposition invariance condition stated by Harris. ${ }^{21}$ This condition implies that the slope of the $V$ iso-lines at the $C=0$ axis must be fixed and equal to 2 . Peter's representation of $V$ data from Orinda study does not fully comply with this requirement. This somewhat restricts the utility of this representation, but we have still chosen to keep this data for two reasons. First, because contrary to what happened in other studies such as Pincus's, ${ }^{9}$ the subject accommodation was not blocked; therefore, in Peters's cohort the subjects may compensate positive defocus by the use of accommodation (this can be readily seen in the plots of Peters's work ${ }^{10}$ ). Second, although Peters's representation of $V$ may be inaccurate at some places, the source for his data, the large and thorough Orinda study, should provide overall meaningful information. In addition, the proposed model is mathematically invariant under transposition, so fitting data obtained from Peter's representation will not alter this property. Further, out of the three age groups created by Peters, we have chosen the third one (45- to 55-years old), as the representation of this group presents the smallest violation of transposition invariance.

Another fact about Peters's data is that there is no information about pupil size. Therefore, if we try to directly adjust our model to those data, we would not be able to distinguish the effect of pupil diameter and that of the parameter $K$, as they are highly correlated according to Eq. (10). Indeed, when we tried to fit the full five parameter model to Peters's data, we got overfitting and large error bounds for both $K$ and $D$. To better understand this issue, we conducted different fits of Peters's data, limiting the fitting parameters to $D, q, A_{\max }$, and $V_{b c}$, using in each fit a different value of $K$ within the interval [0,1], which according to previous work ${ }^{11}$ is where this parameter should lay.

In Fig. 4 we plotted, as a function of $K$, the fitting coefficients $\left\{V_{b c}, D, q, A_{m}\right\}$ and the goodness-of-fit indicators RMSE and AIC. We observe that as $K$ grows from 0.1 to 0.4 , both RSME and AIC get smaller, so the goodness of the fit improves. Also, in this interval, the pupil size is limited by the upper bound set in the fitting algorithm $(8 \mathrm{~mm})$. For $K$ ranging from 0.4 to 1 , 
Table 3 Values of the goodness-of-fit coefficients obtained after fitting the proposed model and those of Blendowske's and Raasch's to Holladay et al.'s acuity data separately for each value of pupil diameter. Legend: $R_{\mathrm{adj}}^{2}$, adjusted $R^{2}$ coefficient; RMSE, root mean square error of the residuals; AIC, Akaike information coefficient; AICc, Akaike information coefficient corrected; wAIC, weight of the AIC coefficient; BIC, Bayesian information coefficient.

\begin{tabular}{|c|c|c|c|c|c|c|c|}
\hline Model & $D(\mathrm{~mm})$ & $R_{\mathrm{adj}}^{2}$ & RMSE & AIC & $\mathrm{AICc}$ & WAIC & $\mathrm{BIC}$ \\
\hline Proposed & 0.5 & 0.9873 & 0.002 & -61.63 & -53.63 & 0.999 & -61.80 \\
\hline Blendowske's & 0.5 & -280.46 & 0.381 & 7.27 & 8.07 & $1.09 \cdot 10^{-15}$ & 7.21 \\
\hline Raasch's & 0.5 & -148.81 & 0.280 & 4.01 & 12.01 & $5.5 \cdot 10^{-15}$ & 3.85 \\
\hline Proposed & 1 & 0.9956 & 0.018 & -34.29 & -26.29 & 0.999 & -34.45 \\
\hline Blendowske's & 1 & -0.2131 & 0.299 & 3.90 & 4.70 & $5.1 \cdot 10^{-9}$ & 3.85 \\
\hline Raasch's & 1 & -3.1842 & 0.556 & 13.73 & 21.73 & $3.7 \cdot 10^{-11}$ & 13.56 \\
\hline Proposed & 2 & 0.9933 & 0.059 & -17.75 & -9.75 & 0.961 & -17.91 \\
\hline Blendowske's & 2 & 0.9803 & 0.101 & -11.33 & -10.53 & 0.039 & -11.38 \\
\hline Raasch's & 2 & -0.5509 & 0.895 & 21.95 & 29.95 & $2.3 \cdot 10^{-9}$ & 21.79 \\
\hline Proposed & 3 & 0.9957 & 0.051 & -19.71 & -11.71 & 0.999 & -19.87 \\
\hline Blendowske's & 3 & 0.9591 & 0.158 & -5.05 & -4.25 & 0.001 & -5.10 \\
\hline Raasch's & 3 & -1.0215 & 1.111 & 23.42 & 31.42 & $4.3 \cdot 10^{-10}$ & 23.26 \\
\hline Proposed & 5 & 0.9985 & 0.027 & -28.65 & -20.65 & 0.999 & -28.81 \\
\hline Blendowske's & 5 & 0.8375 & 0.283 & 3.11 & 3.91 & $1.3 \cdot 10^{-7}$ & 3.05 \\
\hline Raasch's & 5 & -1.030 & 1.000 & 21.95 & 29.95 & $1.0 \cdot 10^{-11}$ & 21.78 \\
\hline Proposed & 7 & 0.9985 & 0.024 & -30.07 & -22.07 & 0.999 & -30.24 \\
\hline Blendowske's & 7 & 0.7946 & 0.290 & 3.47 & 4.27 & $5.2 \cdot 10^{-8}$ & 3.42 \\
\hline Raasch's & 7 & -1.0127 & 0.909 & 20.61 & 28.61 & $9.8 \cdot 10^{-12}$ & 20.45 \\
\hline
\end{tabular}
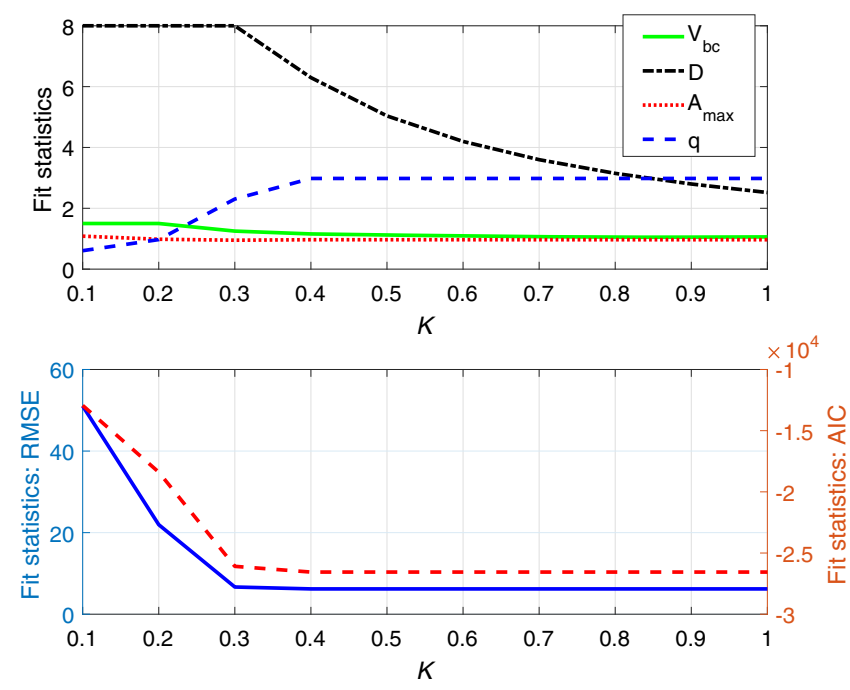

Fig. 4 In the upper plot, we show the evolution of the values of the fitting parameters $V_{b c}$ (continuous line, green in color), pupil diameter (dash-dotted black line), maximum accommodation (dotted line, red in color), diameter (dash-dot line, black in color), and parameter $q$ (dashed line, blue in color). In the lower plot, we have represented the fit statistics RMSE (left vertical axis, blue in color) and AIC (right vertical axis, red in color) against $K$ parameter when fitting Peters's data to our model. we observe that the fitted pupil size satisfies the relation $K D=2.4 \mathrm{~mm}$, with the values for the other fitting parameters and the goodness-of-fit indicators remaining at almost constant values. Therefore, in this case, $K$ and $D$ are not independent but inversely proportional.

Up to this point, we have considered the model as mainly intended to provide individual prediction of visual acuity under different blur situations. Under this approach, we would asses or measure individual parameters as $V_{b c}, q, K$, and $A_{\max }$, and from them, the model would predict $V$ for any given astigmatic blur (normally induced) and pupil size. Peters's data require a different approach, as he used the uncorrected visual acuity measured to a large population sample (around 2000 people in each age group) to obtain the behavior of the average acuity as a function of spherical and astigmatic defocus. We may consider his $V$ plot as the average of many individual $V$ plots obtained by inducing spherical and cylindrical defocus to the same individual with its own parameters $V_{b c}, D, q$, and $A_{\max }$. Hence, by fitting our model to Peters's data, the values we obtain for the fitting parameters are to be understood as "averaged" over a large population sample. Particularly, within this data set, $K$ no longer has individual meaning. As individual maximum acuity is lost, the term $\mathrm{MAR}_{0}$ becomes less relevant and $K$ couples with $D$ in the product $K D$ appearing in the second term of Eq. (5). 


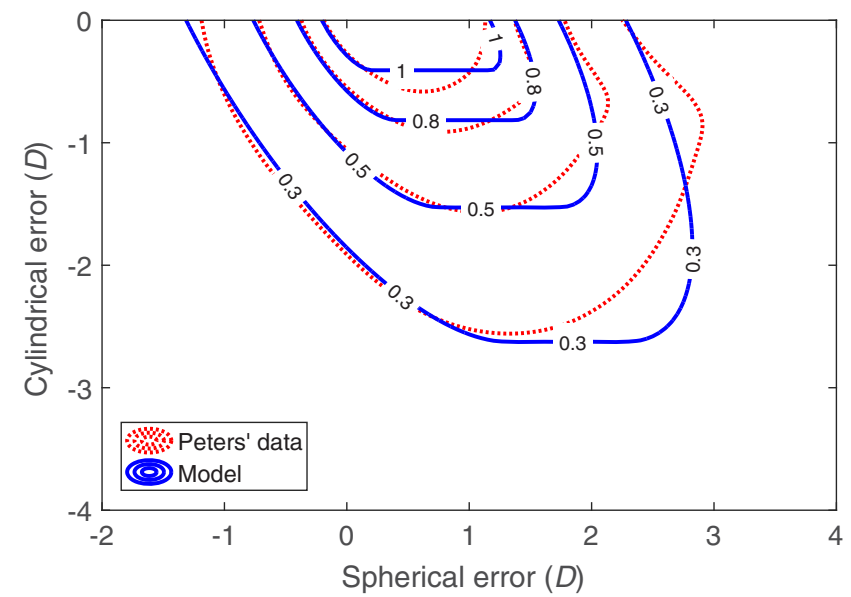

Fig. 5 Contour plot of visual acuity against spherical and cylindrical error. The dashed line represents the values given by Peters for the group of individuals whose age was between 45 and 55 years old (group III in Ref. 10) while the solid line represents the direct fitting of the proposed model to those data.

According to the previous discussion, we can choose any value of $\mathrm{K}$ in the interval $[0.4,1]$. In particular, the value $K=$ 0.8 corresponds to an optimum pupil size of $3 \mathrm{~mm}$. For these values the comparison between Peters Group III $^{10}$ and the predicted acuity is shown in Fig. 4. The group III was formed by the patients with ages between 45 and 55 years old. The resulting fitting parameters are $V_{b c}=1.05 \pm 0.01, D=3.15 \pm 0.01 \mathrm{~mm}$, $q=3.0 \pm 0.1$, and $A_{m}=0.966 \pm 0.004 D$. The contour plots are depicted in Fig. 5. There is a remarkably good agreement in the myopic region and overall good agreement in the hyperopic region. The biggest differences happens at the region with medium hyperopia with moderate cylinder, but even though the iso-lines from the model and the experimental data get more separated here, the actual differences in visual acuity are small, as in this region $V$ is around 0.3 and decreases slowly with both spherical and cylindrical defocus.

These results can be improved by using the averaged nature of Peters's data. To do so, for each $(S, C)$ pair, we generated a set of $N$ random values of the four fitting parameters $\left\{V_{b c}, D, q, A_{m}\right\}$, using a Gaussian random number generator. In this way, we have an $N \times 4$ matrix with rows representing a random state of the fitting parameters $\left\{V_{b c_{j}}, D_{j}, q_{j}\right.$, $\left.A_{\max _{j}}\right\}_{j=1,2, \ldots, N}$, and we computed the visual acuity corresponding to each of those states, $V_{j}=f_{V}\left(S, C, V_{b c_{j}}, D_{j}, q_{j}, A_{\max _{j}}\right)$, where $f_{V}$ stands for the function described in Eq. (10). Finally, we computed the average value of the visual acuity $\left\langle V_{j}\right\rangle$ which depends on the spherical and cylindrical errors and also, on the mean and standard deviation of each fit parameter used in the Gaussian random number generator. To simplify the fitting problem, we used fixed values for the standard deviations of the randomly generated parameters, so the average value of the visual acuity obtained for each $(S, C)$ pair only depends on the set of mean values $\left\{\left\langle V_{b c}\right\rangle,\langle D\rangle,\langle q\rangle,\left\langle A_{\max }\right\rangle\right\}$, which are the fitting parameters of our problem.

The results of this averaging approach are shown in Fig. 6 . Compared to the contour plots of Fig. 5, we obtained a better fit in the region of small hyperopia with cylinder $\sim 1 D$, without losing fit quality in the other zones. As it happened in the previous fit without averaging, the fit results are better in the zone of myopic defocus. Further, if the accommodation were not

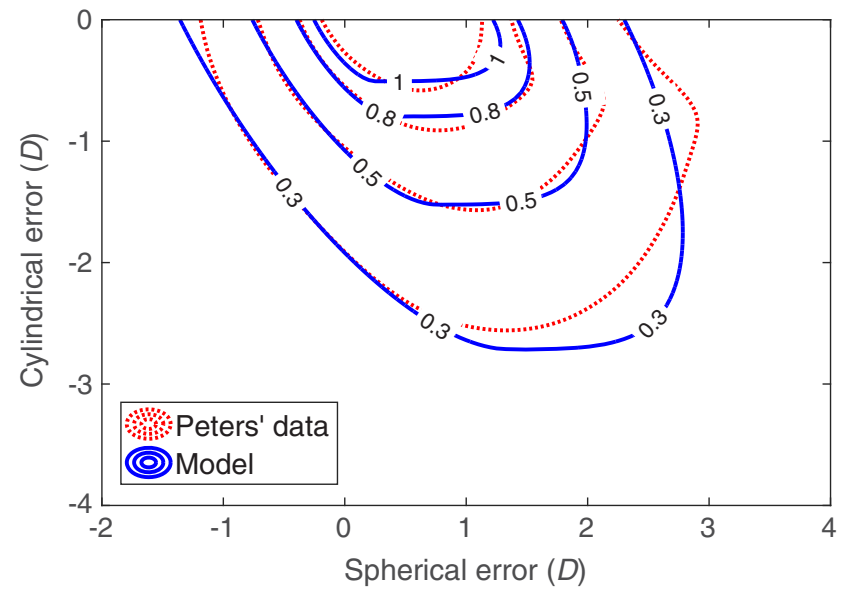

Fig. 6 Contour plot of visual acuity against spherical and cylindrical error. The dashed line represents the values given by Peters for the group of individuals whose age was between 45- and 55-years old (group III in Ref. 10) while the solid line represents the fitting of those data to the proposed model following the statistical averaging procedure described in the text.

included in the model, any fit to hyperopic blur would had been impossible. For the fit with averaging, we have also taken $K=0.8$, and the fitting results are $V_{b c}=1.338 \pm 0.006, D=$ $3.01 \pm 0.01 \mathrm{~mm}, A_{\max }=0.917 \pm 0.005$, and $q=2.46 \pm 0.05$.

\section{Conclusions}

A geometrical model of visual acuity that incorporates nongeometrical effects in a phenomenological way has been proposed. Following the works of Smith, ${ }^{11}$ Raasch, ${ }^{12}$ and Blendowske, ${ }^{14}$ the proposed model incorporates the effects of pupil diameter and astigmatic blur and takes into account the best corrected visual acuity of a given individual. In addition, we have introduced a phenomenological parameter $(q)$, which controls the variation of the acuity for low blur levels, where purely geometrical models fail. This parameter can be associated with the "tolerance to defocus" of a given subject. The proposed model also considers the variation of the best corrected acuity with pupil diameter. Finally, the incorporation of the accommodation in the blur allows for a reasonably good prediction of the visual acuity for hyperopes, individuals with induced positive defocus or in situations of near vision.

These features make the model suitable for designing ophthalmic compensations, particularly for lenses with variable power, which present a considerable induced defocus that will impair the acuity of the user.

The model has been fitted to the data compiled by Holladay et al. ${ }^{5}$ Using the subset of $V$ against pupil diameter when no blur is present, we are able to set $\mathrm{MRA}_{0}(D)$, which describes the behavior of the minimum resolvable angle as a function of the pupil diameter. This information is used to fit the model so that it is able to accurately predict the acuity for any value of blur and pupil diameter for all the remaining data of Holladay et al.'s set. In this fit, all the model parameters seem to have statistical significance, and the values of the Bayesian statistics coefficient AIC and BIC, together with the low error levels in the fitted parameters, indicate that no overfitting is present. Moreover, the proposed model compares favorably with other published models such as the ones by Smith, ${ }^{11}$ Raasch, ${ }^{12}$ and Blendowske. $^{14}$ 
The suitability of the proposed model to fit the visual acuity measurements reported by Peters over a large population has also been studied. In this case, there is no point to speak of individual parameters, so the obtained values should be understood as average values extended over the sample.

For both types of experimental data (Holladay et al.'s and Peters's), the results show the ability of the model to describe the dependence of the visual acuity with the refractive error, including the effect of pupil diameter (when available) and that of accommodation. This could be useful in designing ophthalmic compensations or when a simple modeling of the human visual system is requested as, for example, in problems of visual ergonomic, visual test design, evaluation of ocular compensation techniques, and optical instrumentation.

\section{Disclosures}

The authors of the manuscript, Drs. José A. Gómez-Pedrero and José Alonso, manifest no relevant financial interests, no competing interests and no other potential conflicts of interest to disclose in this work.

\section{Acknowledgments}

The authors wish to thank the economic support for this work given by the Spanish Ministry of Economy and Competitiveness (MINECO) through the Grant No. DPI2012-36103.

\section{References}

1. E. Villegas and P. Artal, "Visual acuity and optical parameters in progressive-power lenses," Optom. Vis. Sci. 83(9), 672-681 (2006).

2. S. Bará, T. Mancebo, and E. Moreno-Barriuso, "Positioning tolerances for phase plates compensating aberrations of the human eye," Appl. Opt. 39(19), 3413-3420 (2000).

3. F. W. Campbell and D. G. Green, "Optical and retinal factors affecting visual resolution,” J. Physiol. 181, 576-593 (1965).

4. G. E. Legge et al., "Tolerance to visual defocus," J. Opt. Soc. Am. A 4, 851-863 (1987).

5. J. T. Holladay et al., "The relationship of visual acuity, refractive error, and pupil size after radial keratotomy," Arch. Ophthalmol. 109, 70-76 (1991).

6. D. Atchison, G. Smith, and N. Efron, "The effect of pupil size on visual acuity in uncorrected and corrected myopia," Optom. Vis. Sci. 56(5), 315-323 (1979).

7. A. Bradley et al., "Effects of spherical and astigmatic defocus on acuity and contrast: a comparison of three clinical charts," Optom. Vis. Sci. 68, 418-426 (1991).
8. R. A. Applegate et al., "Visual acuity as a function of Zernike mode and level of root mean square error," Optom. Vis. Sci. 80, 97-105 (2003).

9. M. H. Pincus, "Unaided visual acuities correlated with refractive errors: a study," Am. J. Ophthalmol. 29, 853-858 (1946).

10. H. B. Peters, "The relationship between refractive error and visual acuity at three age levels," Am. J. Optom. Arch. Am. Acad. Optom. 38, 194-198 (1961).

11. G. Smith, "Relation between spherical refractive error and visual acuity," Optom. Vis. Sci. 68, 591-598 (1991).

12. T. W. Raasch, "Spherocylindrical refractive errors and visual acuity," Optom. Vis. Sci. 72, 272-275 (1995).

13. L. N. Thibos, W. Wheeler, and D. Horner, "Power vectors: an application of Fourier analysis to the description and statistical analysis of refractive errors," Optom. Vis. Sci. 74, 367-375 (1997).

14. R. Blendowske, "Unaided visual acuity and blur: a simple model," Optom. Vis. Sci. 92, e121-e125 (2015).

15. J. E. Greivenkamp et al., "Visual acuity modeling using optical raytracing of schematic eyes," Am. J. Ophthalmol. 120, 227-240 (1995).

16. O. Nestares, R. Navarro, and B. Antona, "Bayesian model of Snellen visual acuity," J. Opt. Soc. Am. A. 20, 1371-1381 (2003).

17. J. Schwiegerling, Field Guide to Visual and Ophthalmic Optics, SPIE Press, Bellingham, Washington (2004).

18. W. F. Long, "A matrix formalism for decentration problems," Am. J. Optometry Physiol. Opt. 53(1), 27-33 (1976).

19. L. Remon, M. Tornel, and W. D. Furlan, "Visual acuity in simple myopic astigmatism: influence of cylinder axis," Optometry Vision Sci. 83(5), 311-315 (2006).

20. A. N. Spiess and N. Neumeyer, "An evaluation of R2 as an inadequate measure for nonlinear models in pharmacological and biochemical research: a Monte Carlo approach,” BMC Pharmacol. 10, 6 (2010).

21. W. F. Harris, "Invariance of ophthalmic properties under spherocylindrical transposition," Optom. Vis. Sci. 74(6), 459-462 (1997).

José A. Gómez-Pedrero is an assistant professor at the College of Optics and Optometry of the University Complutense of Madrid. He received his MS degree in physics and his $\mathrm{PhD}$ in optics from the University Complutense in 1994 and 1998, respectively. He is the author of more than 30 journal papers. His current research interests include ophthalmic optics, image processing, computer vision, and optical design.

José Alonso is an assistant professor at the College of Optics and Optometry of the University Complutense of Madrid and founder of the company, Indizen Optical Technologies. He received his MS degree and PhD in physics from the University Complutense in 1988 and 1992 , respectively. He is the author of more than 50 journal papers and 12 patents. His current research interests include ophthalmic optics, optical design, and optical manufacturing. 